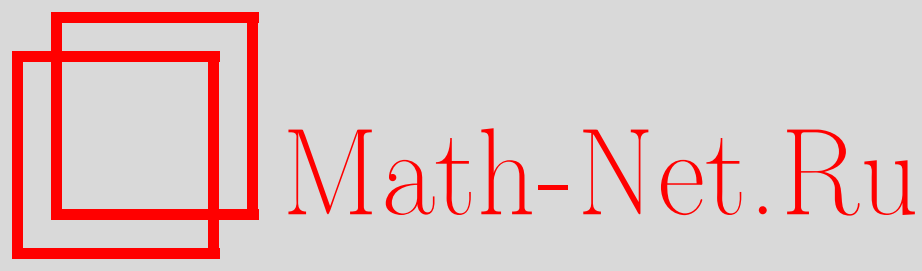

C. М. Ташпулатов, Спектр оператора энергии двухмагнонных систем в изотропной примесной ферромагнитной модели Гейзенберга, ТМФ, 2010, том 164, номер 3, 464-472

DOI: https://doi.org/10.4213/tmf6558

Использование Общероссийского математического портала Math-Net.Ru подразумевает, что вы прочитали и согласны с пользовательским соглашением http://www . mathnet.ru/rus/agreement

Параметры загрузки:

IP : 3.93.64.190

26 апреля 2023 г., $17: 46: 38$

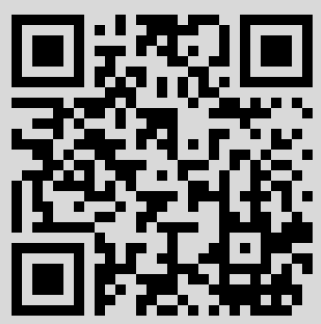




\section{СПЕКТР ОПЕРАТОРА ЭНЕРГИИ ДВУХМАГНОННЫХ СИСТЕМ В ИЗОТРОПНОЙ ПРИМЕСНОЙ ФЕРРОМАГНИТНОЙ МОДЕЛИ ГЕЙЗЕНБЕРГА}

\footnotetext{
Рассматривается двухмагнонная система в изотропной примесной ферромагнитной модели Гейзенберга в $\nu$-мерной решетке $\mathbb{Z}^{\nu}$. Установлено, что существенный спектр системы состоит из объединения не более чем четырех отрезков. Получены нижняя и верхняя оценки для числа трехчастичных связанных состояний системы.
}

Ключевые слова: существенный спектр, дискретный спектр, локальные примесные состояния, связанные состояния, модель Гейзенберга, двухмагнонная система в примесной модели.

\section{1. ВВЕДЕНИЕ}

В настоящей работе рассматривается двухмагнонная система в изотропной примесной ферромагнитной модели Гейзенберга на $\nu$-мерной решетке $\mathbb{Z}^{\nu}$ с взаимодействием ближайших соседей и исследуется существенный и дискретный спектры этой системы.

Опишем сначала модель с конечным числом спинов (см. [1]). Пусть $\Lambda$ - конечное подмножество в $\mathbb{Z}^{\nu}$. Каждой точке $t \in \mathbb{Z}^{\nu}$ сопоставим экземпляр пространства $\mathbb{C}^{2}$ и обозначим его $\mathbb{C}_{t}^{2}$. Рассмотрим гильбертово пространство $\mathscr{H}_{\Lambda}=\bigotimes_{t \in \Lambda} \mathbb{C}_{t}^{2}$.

Пусть $\sigma^{x}, \sigma^{y}, \sigma^{z}$ - матрицы Паули, т. е. $\sigma^{x}=\left(\begin{array}{cc}0 & 1 \\ 1 & 0\end{array}\right), \sigma^{y}=\left(\begin{array}{cc}0 & -i \\ i & 0\end{array}\right), \sigma^{z}=\left(\begin{array}{cc}1 & 0 \\ 0 & -1\end{array}\right)$; положим $\sigma^{ \pm} \equiv\left(\sigma^{x} \pm i \sigma^{y}\right) / 2$.

Для каждого $t \in \Lambda$ обозначим через $\sigma_{t}^{x}, \sigma_{t}^{y}, \sigma_{t}^{z}, \sigma_{t}^{ \pm}$операторы в $\mathscr{H}_{\Lambda}$, действующие соответственно как $\sigma^{x}, \sigma^{y}, \sigma^{z}, \sigma^{ \pm}$на $t$-ю компоненту тензорного произведения и как единичная матрица на остальные компоненты.

Гейзенберговский гамильтониан определяется следующим образом:

$$
H_{\Lambda}=-\frac{1}{2} \sum_{\substack{t \\|t-\beta|=1, t, \beta \in \Lambda}}\left(\sigma_{t}^{z} \sigma_{\beta}^{z}+4 \sigma_{t}^{+} \sigma_{\beta}^{-}\right) .
$$

* Институт ядерной физики АНРУз, Ташкент, Узбекистан.

E-mail: toshpul@mail.ru, toshpul@rambler.ru 
Сумма берется по всем упорядоченным парам $(t, \beta)$ ближайших узлов.

Рассмотрим теперь случай $\Lambda=\mathbb{Z}^{\nu}$. Пусть $\mathscr{H}_{\mathbb{Z}^{\nu}}=\bigotimes_{t \in \mathbb{Z}^{\nu}} \mathbb{C}_{t}^{2}$ - бесконечное тензорное произведение двумерных комплексных гильбертовых пространств $\mathbb{C}^{2}$.

Векторы $e_{1}^{t}=\left(\begin{array}{l}1 \\ 0\end{array}\right), e_{0}^{t}=\left(\begin{array}{l}0 \\ 1\end{array}\right)$ обычно называют состояниями со спином вверх и спином вниз в точке $t$ соответственно. Гильбертовым пространством состояний бесконечного числа спинов по одному в каждой точке $t \in \mathbb{Z}^{\nu}$ как раз и будет пространство $\mathscr{H}_{\mathbb{Z}^{\nu}}=\bigotimes_{t \in \mathbb{Z}^{\nu}} \mathbb{C}_{t}^{2}$.

Множество векторов вида $\bigotimes_{t \in \mathbb{Z}^{\nu}} e_{a_{t}}^{t}$, где $\left\{a_{t}\right\}_{t \in \mathbb{Z}^{\nu}}$ - последовательность нулей и единиц, есть базис в $\mathscr{H}_{\mathbb{Z}^{\nu}}$.

Пусть $\mathscr{H}_{n}$ - линейная оболочка тех векторов $\bigotimes_{t \in \mathbb{Z}^{\nu}} e_{a_{t}}^{t}$ из $\mathscr{H}_{\mathbb{Z}^{\nu}}$, для которых только ровно в $n$ узлах $t \in \mathbb{Z}^{\nu}$ спин направлен вверх, а в остальных узлах спин направлен вниз. Пространство $\mathscr{H}=\bigoplus_{n} \mathscr{H}_{n}$ является фоковским пространством. Определим теперь гамильтониан, действующий в пространстве $\mathscr{H}$.

Непосредственно использовать формулу (1) для определения гамильтониана нельзя, ибо в этом случае сумма будет расходиться. Однако если заменить (1) на

$$
H_{\mathbb{Z}^{\nu}}=-\frac{1}{2} J \sum_{\substack{|t-\beta|=1, t, \beta \in \mathbb{Z}^{\nu}}}\left(\sigma_{t}^{z} \sigma_{\beta}^{z}+4 \sigma_{t}^{+} \sigma_{\beta}^{-}-1\right),
$$

то это определение уже корректно. Используя равенство $\vec{S}_{t}=\vec{\sigma}_{t} / 2$, где $\vec{S}_{t}=$ $\left(S_{t}^{x} ; S_{t}^{y} ; S_{t}^{z}\right), \vec{\sigma}_{t}=\left(\sigma_{t}^{x} ; \sigma_{t}^{y} ; \sigma_{t}^{z}\right)$, гейзенберговский гамильтониан можно записать в виде

$$
H^{\prime}=J \sum_{m, \tau}\left(\vec{S}_{m} \vec{S}_{m+\tau}\right)
$$

Далее мы будем пользоваться операторами $\vec{S}_{m}$ и гамильтониан системы будем описывать на языке операторов $\vec{S}_{m}$.

Гамильтониан рассматриваемой системы имеет вид

$$
H=J \sum_{m, \tau}\left(\vec{S}_{m} \vec{S}_{m+\tau}\right)+\left(J_{0}-J\right) \sum_{\tau}\left(\vec{S}_{0} \vec{S}_{\tau}\right)
$$

где $J<0$ и $J_{0} \neq 0$ - параметры билинейного обменного взаимодействия между атомами и между атомами и примесями соответственно, $\vec{S}_{m}=\left(S_{m}^{x} ; S_{m}^{y} ; S_{m}^{z}\right)$ - оператор атомного спина величины $s=1 / 2$ в узле $m$, а по $\tau$ ведется суммирование по ближайшим соседям. Гамильтониан (4) действует в симметрическом фоковском пространстве $\mathscr{H}$. Обозначим через $\varphi_{0}$ вектор, называемый вакуумным и однозначно определяемый условиями $S_{m}^{+} \varphi_{0}=0, S_{m}^{z} \varphi_{0}=\varphi_{0} / 2$, где $\left\|\varphi_{0}\right\|=1$. Положим $S_{m}^{ \pm}=S_{m}^{x} \pm i S_{m}^{y}$, где $S_{m}^{-}$и $S_{m}^{+}$- соответственно операторы рождения и уничтожения магнона в узле $m$. Вектор $S_{m}^{-} S_{n}^{-} \varphi_{0}$ описывает состояние системы двух магнонов, находящихся в узлах $m$ и $n$, со значением спина $s=1 / 2$. Векторы $S_{m}^{-} S_{n}^{-} \varphi_{0}$ образуют ортонормальную систему. Гильбертово пространство, натянутое на эти векторы, обозначим через $\mathscr{H}_{2}$. Оно называется пространством двухмагнонных состояний оператора $H$. Пространство $\mathscr{H}_{2}$ инвариантно относительно оператора $H$. Обозначим через $H_{2}$ сужение оператора $H$ на $\mathscr{H}_{2}$. 
Наша цель состоит в изучении спектра оператора $H_{2}$. Это удобно делать в его квазиимпульсном представлении. Обозначим через $\mathscr{F}$ преобразование Фурье:

$$
\mathscr{F}: l_{2}\left(\mathbb{Z}^{\nu} \times \mathbb{Z}^{\nu}\right) \rightarrow L_{2}\left(T^{\nu} \times T^{\nu}\right) .
$$

Здесь $T^{\nu}-\nu$-мерный тор, снабженный нормированной мерой Лебега $d \lambda: \lambda\left(T^{\nu}\right)=1$.

Оператор $H_{2}$ в квазиимпульсном представлении в пространстве $L_{2}\left(T^{\nu} \times T^{\nu}\right)$ действует по формуле

$$
\begin{aligned}
& \left(\widetilde{H}_{2} f\right)(x ; y)=\left[-4 J \nu+2 J \sum_{i=1}^{\nu}\left(\cos x_{i}+\cos y_{i}\right)\right] f(x ; y)- \\
& \quad-\varepsilon \int_{T^{\nu}}\left[\nu+\sum_{i=1}^{\nu}\left[\cos \left(x_{i}-s_{i}\right)-\cos x_{i}-\cos s_{i}\right]\right] f(s ; y) d s- \\
& \quad-\varepsilon \int_{T^{\nu}}\left[\nu+\sum_{i=1}^{\nu}\left[\cos \left(y_{i}-t_{i}\right)-\cos y_{i}-\cos t_{i}\right]\right] f(x ; t) d t+ \\
& \quad+J \int_{T^{\nu}}\left[4 \nu-2 \sum_{i=1}^{\nu}\left[\cos x_{i}+\cos y_{i}+\cos s_{i}+\cos \left(x_{i}+y_{i}-s_{i}\right)-\right.\right. \\
& \left.\left.\quad-\cos \left(x_{i}-s_{i}\right)-\cos \left(y_{i}-s_{i}\right)\right]\right] f(s ; x+y-s) d s+ \\
& +\varepsilon \int_{T^{\nu}} \int_{T^{\nu}}\left[2 \nu+2 \sum_{i=1}^{\nu}\left[\cos \left(x_{i}-s_{i}\right)+\cos \left(y_{i}-t_{i}\right)+\cos \left(x_{i}+y_{i}-s_{i}-t_{i}\right)\right]-\right. \\
& -\cos t_{i}-\cos s_{i}-\cos x_{i}-\cos y_{i}-\cos \left(x_{i}-s_{i}-t_{i}\right)-\cos \left(y_{i}-s_{i}-t_{i}\right)- \\
& \left.-\cos \left(x_{i}+y_{i}-s_{i}\right)-\cos \left(x_{i}+y_{i}-t_{i}\right)\right] f(s ; t) d s d t
\end{aligned}
$$

где $\varepsilon=J_{0}-J$.

Спектральные свойства рассматриваемого оператора энергии двухмагнонных систем в изотропной примесной ферромагнитной модели Гейзенберга тесно связаны со спектральными свойствами его двухчастичных подсистем. Саму исходную систему обычно называют трехчастичной системой, а отвечающий ей гамильтониан - трехчастичным оператором. Сначала исследуем спектр и локализованные примесные состояния одномагнонных примесных систем, а также спектр и связанные состояния двухмагнонных систем.

\section{2. ОДНОМАГНОННЫЕ ПРИМЕСНЫЕ СОСТОЯНИЯ}

Гамильтониан одномагнонной примесной системы также имеет вид (4). Обозначим через $\mathscr{H}_{1}$ пространство одномагнонных состояний оператора $H$. Ясно, что пространство $\mathscr{H}_{1}$ также является инвариантным относительно оператора $H$. Обозначим через $H_{1}$ сужение оператора $H$ на пространство $\mathscr{H}_{1}$.

Опишем теперь спектр оператора $H_{1}$. Обозначим через $\mathscr{F}$ преобразование Фурье

$$
\mathscr{F}: l_{2}\left(\mathbb{Z}^{\nu}\right) \rightarrow L_{2}\left(T^{\nu}\right)
$$


ПРЕДЛОЖЕНИЕ. Оператор $H_{1}$ в квазиимпульсном представлении в пространстве $L_{2}\left(T^{\nu}\right)$ действует по формуле

$$
\left(\widetilde{H}_{1} f\right)(x)=h(x) f(x)+\int_{T^{\nu}} h_{1}(x ; t) f(t) d t,
$$

¿əe

$$
\begin{gathered}
h(x)=-2 J\left[\nu-\sum_{i=1}^{\nu} \cos x_{i}\right], \\
h_{1}(x ; t)=-2 \varepsilon\left[\nu+\sum_{i=1}^{\nu}\left[\cos \left(x_{i}-t_{i}\right)-\cos x_{i}-\cos t_{i}\right]\right], \\
\varepsilon=J_{0}-J, \quad x, t \in T^{\nu} .
\end{gathered}
$$

Известно, что непрерывный спектр оператора $\widetilde{H}_{1}$ не зависит от функции $h_{1}(x ; t)$ и заполняет весь отрезок $\left[m_{\nu} ; M_{\nu}\right]$, где $m_{\nu}=\min _{x \in T^{\nu}} h(x), M_{\nu}=\max _{x \in T^{\nu}} h(x)$.

ОпрЕДЕЛЕниЕ 1 . Собственная функция $\varphi \in L_{2}\left(T^{\nu}\right)$ оператора $\widetilde{H}_{1}$, отвечающая собственному значению $z$, называется локалъным примесным состоянием (ЛПС) оператора $\widetilde{H}_{1}$, а величина $z$ - энергией этого состояния.

Положим

$$
\Delta_{\nu}(z)=\left(1-2 \varepsilon J_{\nu, 1}(z)\right)\left(1-2 \varepsilon J_{\nu, 2}(z)\right)^{\nu}\left(1-\varepsilon J_{\nu, 3}(z)\right)^{\nu-1},
$$

где

$$
\begin{aligned}
& J_{\nu, 1}(z)=\int_{T^{\nu}} \frac{\left(1-\cos s_{1}\right)\left(\nu-\sum_{i=1}^{\nu} \cos s_{i}\right) d s_{1} d s_{2} \ldots d s_{\nu}}{h\left(s_{1} ; s_{2} ; \ldots ; s_{\nu}\right)-z}, \\
& J_{\nu, 2}(z)=\int_{T^{\nu}} \frac{\sin ^{2} s_{1} d s_{1} d s_{2} \ldots d s_{\nu}}{h\left(s_{1} ; s_{2} ; \ldots ; s_{\nu}\right)-z} \\
& J_{\nu, 3}(z)=\int_{T^{\nu}} \frac{\left(\cos s_{1}-\cos s_{2}\right)^{2} d s_{1} d s_{2} \ldots d s_{\nu}}{h\left(s_{1} ; s_{2} ; \ldots ; s_{\nu}\right)-z} .
\end{aligned}
$$

Лемма. Число $z_{0} \notin\left[m_{\nu} ; M_{\nu}\right]$ является собственным значением оператора $\widetilde{H}_{1}$ тогда и только тогда, когда оно является нулем функиии $\Delta_{\nu}(z)$.

ДокАзАтЕльство. Уравнение для собственных значений является интегральным уравнением с вырожденным ядром. Поэтому оно эквивалентно системе линейных однородных алгебраических уравнений. Известно, что система линейных однородных алгебраических уравнений имеет нетривиальное решение тогда и только тогда, когда детерминант системы равен нулю. Учитывая, что функция $h\left(s_{1} ; s_{2} ; \ldots ; s_{\nu}\right)$ является симметрической функцией относительно $s_{i}$ и $s_{j}$, и выполняя соответствующие преобразования, получаем, что детерминант системы имеет вид $\Delta_{\nu}(z)$.

Уравнение $\Delta_{\nu}(z)=0$ имеет место, если имеет место хотя бы одно из следующих уравнений:

$$
\begin{aligned}
1-2 \varepsilon J_{\nu, 1}(z) & =0, \\
\left(1-2 \varepsilon J_{\nu, 2}(z)\right)^{\nu} & =0, \\
\left(1-\varepsilon J_{\nu, 3}(z)\right)^{\nu-1} & =0 .
\end{aligned}
$$


Поэтому в $\nu$-мерном случае возможны следующие случаи: уравнение $\Delta_{\nu}(z)=0$ может не иметь ни одного решения, может иметь одно, два или три решения; эти решения соответственно невырожденны, $\nu$-кратно вырожденны и $(\nu-1)$-кратно вырожденны. Мы обозначим эти решения соответственно через $z_{1}, z_{2}, z_{3}$.

В работе [2] исследуется спектр и ЛПС одномагнонных систем в примесной модели Гейзенберга на $\nu$-мерной решетке $\mathbb{Z}^{\nu}$, при этом рассматривается как случай ферромагнетика с ферромагнитной примесью, так и случай ферромагнетика с антиферромагнитной примесью. Показано, что в $\nu$-мерном случае система имеет не более трех типов (без учета кратности вырождений их энергии) ЛПС, и найдено условие существования этих состояний. Определено местоположение их энергии относительно непрерывного спектра системы. Мы будем использовать результаты работы [2].

\section{3. ДВУХМАГНОННОЕ СОСТОЯНИЕ}

Гамильтониан двухмагнонной системы имеет вид (3), где $J<0$ - параметр билинейного обменного взаимодействия между ближайшими атомами решетки. Гамильтониан (3) действует в симметрическом фоковском пространстве $\mathscr{H}$. Через $\mathscr{H}_{2}$ обозначим пространство двухмагнонных состояний оператора (3). Оно инвариантно относительно оператора $H^{\prime}$. Обозначим через $H_{2}^{\prime}$ сужение оператора $H^{\prime}$ на $\mathscr{H}_{2}$.

Пусть $\widetilde{\mathscr{H}_{2}}=\mathscr{F} \mathscr{H}_{2}$. Оператор $H_{2}^{\prime}$ в квазиимпульсном представлении в пространстве $L_{2}\left(T^{\nu} \times T^{\nu}\right)$ действует по формуле

$$
\left(\widetilde{H}_{2}^{\prime} f\right)(x ; y)=h(x ; y) f(x ; y)-\int_{T^{\nu}} h_{1}(x ; y ; s) f(s ; x+y-s) d s,
$$

где

$$
\begin{aligned}
h_{1}(x ; y ; s) & =h(x ; y)+h_{2}(x ; y ; s), \\
h(x ; y) & =-4 \nu J+2 J \sum_{i=1}^{\nu}\left(\cos x_{i}+\cos y_{i}\right), \\
h_{2}(x ; y ; s) & =2 J \sum_{i=1}^{\nu}\left[\cos s_{i}+\cos \left(x_{i}+y_{i}-s_{i}\right)-\cos \left(x_{i}-s_{i}\right)-\cos \left(y_{i}-s_{i}\right)\right] .
\end{aligned}
$$

Пусть фиксирован полный квазиимпульс системы $x+y=\Lambda$. Обозначим через $L_{2}\left(\Gamma_{\Lambda}\right)$ пространство функций, квадратично интегрируемых по многообразию $\Gamma_{\Lambda}=\{(x ; y): x+y=\Lambda\}$. Известно [3], что оператор $\widetilde{H}_{2}^{\prime}$ и пространство $\widetilde{\mathscr{H}}_{2}$ можно разложить в прямой интеграл

$$
\widetilde{H}_{2}^{\prime}=\bigoplus \int_{T^{\nu}} \widetilde{H}_{2 \Lambda}^{\prime} d \Lambda, \quad \widetilde{\mathscr{H}}_{2}=\bigoplus \int_{T^{\nu}} \widetilde{\mathscr{H}}_{2 \Lambda} d \Lambda
$$

операторов $\widetilde{H}_{2 \Lambda}^{\prime}$ и пространств $\widetilde{\mathscr{H}}_{2 \Lambda}=L_{2}\left(\Gamma_{\Lambda}\right)$ так, что пространства $\widetilde{\mathscr{H}}_{2 \Lambda}$ окажутся инвариантными относительно операторов $\widetilde{H}_{2 \Lambda}^{\prime}$, а операторы $\widetilde{H}_{2 \Lambda}^{\prime}$ в пространстве $\widetilde{\mathscr{H}}_{2 \Lambda}$ действуют по формуле

$$
\left(\widetilde{H}_{2 \Lambda}^{\prime} f_{\Lambda}\right)(x)=h_{\Lambda}(x) f_{\Lambda}(x)-\int_{T^{\nu}} h_{1 \Lambda}(x ; t) f_{\Lambda}(t) d t,
$$

где $h_{\Lambda}(x)=h(x ; \Lambda-x), h_{1 \Lambda}(x ; t)=h_{1}(x ; \Lambda-x ; t), f_{\Lambda}(x)=f(x ; \Lambda-x)$.

Известно, что непрерывный спектр оператора $\widetilde{H}_{2 \Lambda}^{\prime}$ не зависит от функций $h_{1 \Lambda}(x ; t)$ и состоит из отрезков $G_{\Lambda}=\left[m_{\Lambda} ; M_{\Lambda}\right]$, где $m_{\Lambda}=\inf _{x} h_{\Lambda}(x)$ и $M_{\Lambda}=\sup _{x} h_{\Lambda}(x)$. 
ОПрЕДЕЛЕНИЕ 2. Собственная функция $\varphi_{\Lambda} \in L_{2}\left(T^{\nu} \times T^{\nu}\right)$ оператора $\widetilde{H}_{2}^{\prime}$, отвечающая собственному значению $z_{\Lambda}$, называется связанным состоянием (CC) оператора $\widetilde{H}_{2}^{\prime}$, а величина $z_{\Lambda}$ - энергией этого состояния.

Спектр и СС оператора энергии двухмагнонных систем были изучены в работах [4], [5], где показано, что такие системы в $\nu$-мерной решетке имеют не более чем $2 \nu+1 \mathrm{CC}$ со значениями энергии, лежащими вне области $G_{\Lambda}-$ непрерывного спектра системы.

\section{4. СТРУКТУРА СУЩЕСТВЕННОГО СПЕКТРА ТРЕХЧАСТИЧНОЙ СИСТЕМЫ}

Сначала определим структуру существенного спектра рассматриваемой трехчастичной системы, состоящей из двух магнонов и примесного спина, а затем оценим количество трехчастичных СС этой системы. Сравнивая формулы (??) и (6) и используя тензорные произведения гильбертовых пространств и тензорные произведения операторов в гильбертовых пространствах [6], можно убедиться, что оператор $\widetilde{H}_{2}$ можно представить в виде $\widetilde{H}_{2}=\widetilde{H}_{1} \otimes E+E \otimes \widetilde{H}_{1}+K_{1}+K_{2}$, где $E$ - единичный оператор в пространстве $\widetilde{\mathscr{H}}_{1}$, а операторы $K_{1}, K_{2}$ являются интегральными операторами следующего вида:

$$
\begin{aligned}
& \left(K_{1} f\right)(x ; y)=J \int_{T^{\nu}} k_{1}(x ; y ; s) f(s ; x+y-s) d s, \\
& \left(K_{2} f\right)(x ; y)=\varepsilon \int_{T^{\nu}} \int_{T^{\nu}} k_{2}(x ; y ; s ; t) f(s ; t) d s d t .
\end{aligned}
$$

Ядра этих операторов соответственно имеют вид

$$
\begin{aligned}
k_{1}(x ; y ; s)= & 4 \nu-\sum_{i=1}^{\nu}\left[\cos x_{i}+\cos y_{i}+\cos s_{i}+\cos \left(x_{i}+y_{i}-s_{i}\right)-\right. \\
& \left.-\cos \left(x_{i}-s_{i}\right)-\cos \left(y_{i}-s_{i}\right)\right], \\
k_{2}(x ; y ; s ; t)= & 2 \nu+2 \sum_{i=1}^{\nu}\left\{\left[\cos \left(x_{i}-s_{i}\right)+\cos \left(y_{i}-t_{i}\right)+\cos \left(x_{i}+y_{i}-s_{i}-t_{i}\right)\right]-\right. \\
& -\cos t_{i}-\cos s_{i}-\cos x_{i}-\cos y_{i}-\cos \left(x_{i}-s_{i}-t_{i}\right)-\cos \left(y_{i}-s_{i}-t_{i}\right)- \\
& \left.-\cos \left(x_{i}+y_{i}-t_{i}\right)-\cos \left(x_{i}+y_{i}-s_{i}\right)\right\},
\end{aligned}
$$

где $\varepsilon=J_{0}-J$.

Как уже отмечалось (см. раздел 3), при фиксированном полном квазиимпульсе двухмагнонной подсистемы $x+y=\Lambda$ оператор $H_{2}^{\prime}$ и пространство $\mathscr{H}_{2}$ можно разложить в прямой интеграл:

$$
\widetilde{H}_{2}^{\prime}=\bigoplus \int_{T^{\nu}} \widetilde{H}_{2 \Lambda}^{\prime} d \Lambda, \quad \widetilde{\mathscr{H}}_{2}=\bigoplus \int_{T^{\nu}} \mathscr{H}_{2 \Lambda} d \Lambda,
$$

так что после этого разложения операторы $K_{1 \Lambda}$ становятся компактными операторами.

Из выражений для ядер операторов $K_{1}$ и $K_{2}$ видно, что операторы $K_{1 \Lambda}$ и $K_{2}$ являются операторами конечного ранга, т. е. конечномерными операторами. Поэтому существенные спектры оператора $\widetilde{H}_{2}$ и оператора $\widetilde{H}_{1} \otimes E+E \otimes \widetilde{H}_{1}$ совпадают. 
Простая проверка показывает, что спектр оператора $\widetilde{H}_{1}$ не зависит от $\Lambda$, т. еот $\lambda$ и $\mu$. Спектр оператора $A \otimes E+E \otimes B$, где $A$ и $B$ - плотно определенные ограниченные линейные операторы, был изучен в работах [7]-[9]. В этих работах даны явные формулы, выражающие $\sigma_{\mathrm{ess}}(A \otimes E+E \otimes B)$ и $\sigma_{\mathrm{disc}}(A \otimes E+E \otimes B)$ через $\sigma(A)$, $\sigma_{\text {disc }}(A), \sigma(B)$ и $\sigma_{\text {disc }}(B)$ :

$$
\begin{aligned}
\sigma_{\text {disc }}(A \otimes E+E \otimes B)= & \left\{\sigma(A) \backslash \sigma_{\text {ess }}(A)+\sigma(B) \backslash \sigma_{\text {ess }}(B)\right\} \backslash\left\{\left(\sigma_{\text {ess }}(A)+\sigma(B)\right) \cup\right. \\
& \left.\cup\left(\sigma(A)+\sigma_{\text {ess }}(B)\right)\right\}, \\
\sigma_{\text {ess }}(A \otimes E+E \otimes B)= & \left(\sigma_{\text {ess }}(A)+\sigma(B)\right) \cup\left(\sigma(A)+\sigma_{\text {ess }}(B)\right) .
\end{aligned}
$$

Ясно, что $\sigma(A \otimes E+E \otimes B)=\{\lambda+\mu: \lambda \in \sigma(A), \mu \in \sigma(B)\}$. Из результатов работы [2] видно, что спектр оператора $\widetilde{H}_{1}$ состоит из непрерывного спектра и не более чем трех собственных значений, которые соответственно невырожденны, $\nu$-кратно и $(\nu-1)$-кратно вырожденны.

Следующие теоремы описывают структуру существенного спектра оператора $\widetilde{H}_{1} \otimes E+E \otimes \widetilde{H}_{1}$.

Теорема 1. Если $\nu=1$ и $0<\varepsilon \leqslant-J$, то существенный спектр оператора $\widetilde{H}_{2}$ состоит из единственного отрезка $\sigma_{\mathrm{ess}}\left(\widetilde{H}_{2}\right)=[0 ;-8 J]$, и для числа трехчастичнъх CС имеет место соотношение $0 \leqslant N \leqslant 12$.

Теорема 2. Если $\nu=1 u J \leqslant \varepsilon<0$, то существенный спектр оператора $\widetilde{H}_{2}$ состоит из обгединения двух отрезков: $\sigma_{\mathrm{ess}}\left(\widetilde{H}_{2}\right)=[0 ;-8 J] \cup\left[z_{1} ;-4 J+z_{1}\right]$, и для числа трехчастичных СС имеет место соотночение $1 \leqslant N \leqslant 13$.

ТеОрема 3. Если $\nu=1 u \varepsilon>-J$ или $\varepsilon<J$, то существенный спектр оператоpa $\widetilde{H}_{2}$ состоит из обгединения трех отрезков: $\sigma_{\mathrm{ess}}\left(\widetilde{H}_{2}\right)=[0 ;-8 J] \cup\left[z_{1} ;-4 J+z_{1}\right] \cup$ $\left[z_{2} ;-4 J+z_{2}\right]$, и для числа трехчастичных CC имеет место соотношение $3 \leqslant$ $N \leqslant 15$.

ТеОрема 4. Если $\nu=2 u 0<\varepsilon \leqslant-J$ или $J / 3<\varepsilon<0$, то существенный спектр оператора $\widetilde{H}_{2}$ состоит из единственного отрезка $\sigma_{\mathrm{ess}}\left(\widetilde{H}_{2}\right)=[0 ;-16 J]$, и для числа трехчастичных CC имеет место соотношение $0 \leqslant N \leqslant 22$.

Tеорема 5. Если $\nu=2 u-J<\varepsilon \leqslant-100 J / 27$ или $25 J / 9<\varepsilon<0$, mо существенный спектр оператора $\widetilde{H}_{2}$ состоит из обгединения двух отрезков: $\sigma_{\mathrm{ess}}\left(\widetilde{H}_{2}\right)=$ $[0 ;-16 J] \cup\left[z_{1} ;-8 J+z_{1}\right]$, и для числа трехчастичных CC имеет место соотношение $1 \leqslant N \leqslant 23$.

Теорема 6. Если $\nu=2 u-100 J / 27<\varepsilon \leqslant-25 J / 9$ или $100 J / 27<\varepsilon \leqslant 25 J / 9$, то существенный спектр оператора $\widetilde{H}_{2}$ состоит из обгединения трех отрезков: $\sigma_{\text {ess }}\left(\widetilde{H}_{2}\right)=[0 ;-16 J] \cup\left[z_{1} ;-8 J+z_{1}\right] \cup\left[z_{2} ;-8 J+z_{2}\right]$, и для числа трехчастичных CC имеет место соотношение $3 \leqslant N \leqslant 25$.

Теорема 7. Если $\nu=2 u \varepsilon>-25 \mathrm{~J} / 9$ или $\varepsilon \leqslant 100 \mathrm{~J} / 27$, то существенный спектр оператора $\widetilde{H}_{2}$ состоит из обгединения четырех отрезков: $\sigma_{\mathrm{ess}}\left(\widetilde{H}_{2}\right)=$ $[0 ;-16 J] \cup\left[z_{1} ;-8 J+z_{1}\right] \cup\left[z_{2} ;-8 J+z_{2}\right] \cup\left[z_{3} ;-8 J+z_{3}\right]$, и для числа трехчастичных СС имеет место соотношение $6 \leqslant N \leqslant 28$. 
ТеОРема 8. Если $\nu=3 u 0<\varepsilon<-J$ или $J / 3<\varepsilon<0$, то существенный спектр оператора $\widetilde{H}_{2}$ состоит из единственного отрезка $\sigma_{\mathrm{ess}}\left(\widetilde{H}_{2}\right)=[0 ;-24 J]$, и для числа трехчастичных СС имеет место соотношение $0 \leqslant N \leqslant 32$.

ДоказАтельство. Из теоремы 3 (п. А) работы [2] видно, что для $\nu=3$ и $0<$ $\varepsilon<-J$ или $J / 3<\varepsilon<0$ оператор $\widetilde{H}_{1}$ не имеет собственных значений, лежащих вне области непрерывного спектра оператора $\widetilde{H}_{1}$, а непрерывный спектр оператора $\widetilde{H}_{1}$ состоит из отрезка $[0 ;-12 J]$, поэтому существенный спектр оператора $\widetilde{H}_{2}$ состоит из отрезка $[0 ;-24 J]$, т. е. $\sigma_{\text {ess }}\left(\widetilde{H}_{2}\right)=\sigma_{\text {ess }}\left(\widetilde{H}_{1}\right)+\sigma\left(\widetilde{H}_{1}\right)=\left\{\lambda+\mu: \lambda \in \sigma_{\text {ess }}\left(\widetilde{H}_{1}\right), \mu \in\right.$ $\left.\sigma\left(\widetilde{H}_{1}\right)\right\}=[0 ;-24 J]$. Поэтому оператор $\widetilde{H}_{1} \otimes E+E \otimes \widetilde{H}_{1}$ вне отрезка $[0 ;-24 J]$ не имеет собственных значений, а операторы $\left(K_{1 \Lambda} f\right)(x)=\left(K_{1 \Lambda} f\right)(x ; \Lambda-x)$ и $\left(K_{2} f\right)(x ; y)$ являются операторами рангов 7 и 25 соответственно. Следовательно, в этом случае число трехчастичных СС $N \leqslant 32$.

Теоремы 1-7 доказываются аналогично.

Введем обозначения:

$$
a=\int_{T^{3}} \frac{\sin ^{2} s_{1} d s_{1} d s_{2} d s_{3}}{3-\cos s_{1}-\cos s_{2}-\cos s_{3}}, \quad b=\int_{T^{3}} \frac{\left(\cos s_{1}-\cos s_{2}\right)^{2} d s_{1} d s_{2} d s_{3}}{3-\cos s_{1}-\cos s_{2}-\cos s_{3}} .
$$

Ясно, что $0<a<b<1$ и $2 a<b$.

Tеорема 9. Если $\nu=3 u-J \leqslant \varepsilon<-2 J / b$ или $2 J / b \leqslant \varepsilon \leqslant J / 3$, то существенный спектр оператора $\widetilde{H}_{2}$ состоит из обгединения двух отрезков: $\sigma_{\mathrm{ess}}\left(\widetilde{H}_{2}\right)=$ $[0 ;-24 J] \cup\left[z_{1} ;-12 J+z_{1}\right]$, где $z_{1}$ - решение уравнения (8), и для числа трехчастичных СС имеет место соотношение $1 \leqslant N \leqslant 33$.

ДокАЗАТЕЛЬСТВо. Из теоремы 3 (п. Б) работы [2] видно, что для $\nu=3$ и $2 J / b \leqslant \varepsilon \leqslant J / 3$ или $-J \leqslant \varepsilon<-2 J / b$ оператор $\widetilde{H}_{1}$ имеет единственное собственное значение $z=z_{1}$, лежащее вне области непрерывного спектра оператора $\widetilde{H}_{1}$. Поэтому множество $\sigma_{\text {ess }}\left(\widetilde{H}_{2}\right)=\sigma_{\text {ess }}\left(\widetilde{H}_{1}\right)+\sigma\left(\widetilde{H}_{1}\right)=\left\{\lambda+\mu: \lambda \in \sigma_{\text {ess }}\left(\widetilde{H}_{1}\right), \mu \in \sigma\left(\widetilde{H}_{1}\right)\right\}$ состоит из объединения двух отрезков: $[0 ;-24 J] \cup\left[z_{1} ;-12 J+z_{1}\right]$, т. е. $\sigma_{\text {ess }}\left(\widetilde{H}_{2}\right)=$ $[0 ;-24 J] \cup\left[z_{1} ;-12 J+z_{1}\right]$. Число $2 z_{1}$ является собственным значением для оператора $\widetilde{H}_{1} \otimes E+E \otimes \widetilde{H}_{1}$, и оно лежит вне области существенного спектра оператора $\widetilde{H}_{1} \otimes E+E \otimes \widetilde{H}_{1}$. Поэтому для числа трехчастичных СС имеет место соотношение $1 \leqslant N \leqslant 33$.

Tеорема 10. Если $\nu=3 u-2 J / b \leqslant \varepsilon<-J / a$ uли $J / a \leqslant \varepsilon \leqslant 2 J / b$, mo cyщественный спектр оператора $\widetilde{H}_{2}$ состоит из обгединения трех отрезков: $\sigma_{\mathrm{ess}}\left(\widetilde{H}_{2}\right)=$ $[0 ;-24 J] \cup\left[z_{1} ;-12 J+z_{1}\right] \cup\left[z_{2} ;-12 J+z_{2}\right]$, где $z_{1}$ - решение уравнения (8), а $z_{2}$ решение уравнения (9), и для числа трехчастичных СС имеет место соотношение $3 \leqslant N \leqslant 35$.

ДокАЗАТЕЛЬСтво. Из теоремы 3 (п. В) работы [2] видно, что для $\nu=3$ и $-2 J / b \leqslant \varepsilon \leqslant J / a$ или $-J / a \leqslant \varepsilon \leqslant 2 J / b$ оператор $\widetilde{H}_{1}$ имеет ровно два собственных значения $z_{1}$ и $z_{2}$, лежащих вне области непрерывного спектра оператора $\widetilde{H}_{1}$. Поэтому множество $\sigma_{\text {ess }}\left(\widetilde{H}_{2}\right)=\sigma_{\text {ess }}\left(\widetilde{H}_{1}\right)+\sigma\left(\widetilde{H}_{1}\right)=\left\{\lambda+\mu: \lambda \in \sigma_{\text {ess }}\left(\widetilde{H}_{1}\right), \mu \in \sigma\left(\widetilde{H}_{1}\right)\right\}$ состоит из объединения трех отрезков: $[0 ;-24 J] \cup\left[z_{1} ;-12 J+z_{1}\right] \cup\left[z_{2} ;-12 J+z_{2}\right]$, т. е. $\sigma_{\text {ess }}\left(\widetilde{H}_{2}\right)=[0 ;-24 J] \cup\left[z_{1} ;-12 J+z_{1}\right] \cup\left[z_{2} ;-12 J+z_{2}\right]$. Числа $2 z_{1}, 2 z_{2}$ и $z_{1}+z_{2}$ являются собственными значениями для оператора $\widetilde{H}_{1} \otimes E+E \otimes \widetilde{H}_{1}$, и они лежат 
вне области существенного спектра оператора $\widetilde{H}_{1} \otimes E+E \otimes \widetilde{H}_{1}$. Поэтому для числа трехчастичных СС имеет место соотношение $3 \leqslant N \leqslant 35$.

Теорема 11. Если $\varepsilon \geqslant-J / a$ или $\varepsilon \leqslant J / a$, то существенный спектр оператора $\widetilde{H}_{2}$ состоит из обгединения четырех отрезков: $\sigma_{\mathrm{ess}}\left(\widetilde{H}_{2}\right)=[0 ;-24 J] \cup\left[z_{1} ;-12 J+\right.$ $\left.z_{1}\right] \cup\left[z_{2} ;-12 J+z_{2}\right] \cup\left[z_{3} ;-12 J+z_{3}\right]$, где $z_{1}, z_{2}, z_{3}$ - соответственно решения уравнений (8)-(10), и для числа трехчастичных CС имеет место соотношение $6 \leqslant N \leqslant 38$.

ДокАЗАТЕЛЬСТВо. Из теоремы 3 (п. Г) работы [2] видно, что для $\nu=3$ и $\varepsilon \geqslant-J / a$ или $\varepsilon \leqslant J / a$ оператор $\widetilde{H}_{1}$ имеет ровно три собственных значения $z_{1}, z_{2}$ и $z_{3}$, лежащих вне области непрерывного спектра оператора $\widetilde{H}_{1}$. Поэтому множество $\sigma_{\mathrm{ess}}\left(\widetilde{H}_{2}\right)=\sigma_{\mathrm{ess}}\left(\widetilde{H}_{1}\right)+\sigma\left(\widetilde{H}_{1}\right)=\left\{\lambda+\mu: \lambda \in \sigma_{\mathrm{ess}}\left(\widetilde{H}_{1}\right), \mu \in \sigma\left(\widetilde{H}_{1}\right)\right\}$ состоит из объединения четырех отрезков: $[0 ;-24 J] \cup\left[z_{1} ;-12 J+z_{1}\right] \cup\left[z_{2} ;-12 J+z_{2}\right] \cup\left[z_{3} ;-12 J+z_{3}\right]$, т. е. $\sigma_{\text {ess }}\left(\widetilde{H}_{2}\right)=[0 ;-24 J] \cup\left[z_{1} ;-12 J+z_{1}\right] \cup\left[z_{2} ;-12 J+z_{2}\right] \cup\left[z_{3} ;-12 J+z_{3}\right]$. Числа $2 z_{1}$, $2 z_{2}, 2 z_{3}, z_{1}+z_{2}, z_{1}+z_{3}$ и $z_{2}+z_{3}$ являются собственными значениями для оператора $\widetilde{H}_{1} \otimes E+E \otimes \widetilde{H}_{1}$, и они лежат вне области существенного спектра оператора $\widetilde{H}_{1} \otimes E+E \otimes \widetilde{H}_{1}$. Поэтому для числа трехчастичных СС имеет место соотношение $6 \leqslant N \leqslant 38$.

Отметим, что структура существенного спектра рассматриваемого оператора в трехмерном случае была исследована в работе автора [10], где была показана конечность дискретного спектра системы в краях существенного спектра.

Результаты, относящиеся к случаям размерности $\nu=1$ и $\nu=2$, являются новыми. Для случая $\nu=3$ конечность дискретного спектра установлена в [10], в настоящей работе даны новые оценки для числа $N$ трехчастичных СС.

Благодарности. Автор выражает благодарность профессору В.И. Чилину за полезные обсуждения. Автор также признателен Р. А. Минлосу за замечания, которые способствовали улучшению настоящей работы.

\section{Список литературы}

[1] М. Рид, Б. Саймон, Методь современной математической физики. т. 3, Мир, М., 1982.

[2] С. М. Ташпулатов, ТМФ, 126:3 (2001), 482-488.

[3] М. А. Наймарк, Нормированнъе кольца, Наука, М., 1968.

[4] M. Wortis, Phys. Rev., 132:1 (1963), 85-97.

[5] С. М. Ташпулатов, ТМФ, 107:1 (1996), 155-161.

[6] М. Рид, Б. Саймон, Методъ современной математической физики. т. 1: Функииональный анализ, Мир, М., 1977.

[7] T. Ichinose, Trans. Amer. Math. Soc., 235 (1978), 75-113.

[8] T. Ichinose, Trans. Amer. Math. Soc., 237 (1978), 223-254.

[9] T. Ichinose, "On the spectral properties of tensor products of linear operators in Banach spaces", Spectral Theory, Banach Center Publ., 8, eds. W. Żelazko, PWN, Warsaw, 1982, 294-300.

[10] С. М. Ташпулатов, ТМФ, 162:2 (2010), 227-242. 